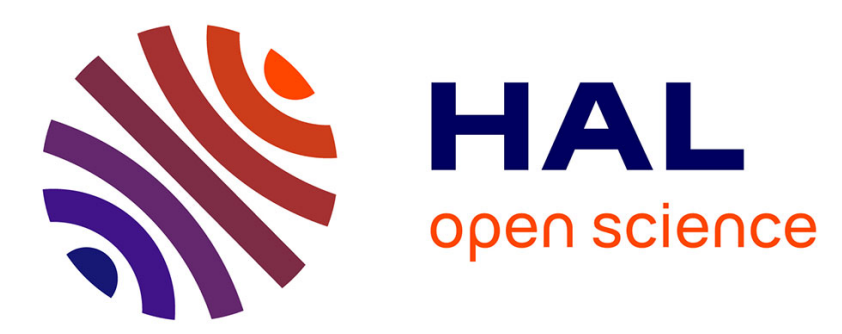

\title{
Generalised Serre-Green-Naghdi equations for open channel and for natural river hydraulics
}

\author{
Mohamed Ali Debyaoui, Mehmet Ersoy
}

\section{To cite this version:}

Mohamed Ali Debyaoui, Mehmet Ersoy. Generalised Serre-Green-Naghdi equations for open channel and for natural river hydraulics. Asymptotic Analysis, inPress, 10.3233/ASY-201647 . hal-02444355

\section{HAL Id: hal-02444355 \\ https://hal.science/hal-02444355}

Submitted on 17 Jan 2020

HAL is a multi-disciplinary open access archive for the deposit and dissemination of scientific research documents, whether they are published or not. The documents may come from teaching and research institutions in France or abroad, or from public or private research centers.
L'archive ouverte pluridisciplinaire HAL, est destinée au dépôt et à la diffusion de documents scientifiques de niveau recherche, publiés ou non, émanant des établissements d'enseignement et de recherche français ou étrangers, des laboratoires publics ou privés. 


\title{
Generalised Serre-Green-Naghdi equations for open channel and for natural river hydraulics.
}

\author{
Mohamed Ali Debyaour ${ }^{* 1}$ and Mehmet Ersoy ${ }^{\dagger 1}$ \\ ${ }^{1}$ Université de Toulon, IMATH EA 2134, 83957 La Garde, France.
}

January 17, 2020

\begin{abstract}
In this paper, we present a new non-linear dispersive model for open channel and river flows. These equations are the second order shallow water approximation of the section-averaged (three-dimensional) incompressible and irrotational Euler system. This new asymptotic model generalises the well-known one-dimensional Serre-GreenNaghdi (SGN) equations for rectangular section on uneven bottom to arbitrary channel/river section.
\end{abstract}

Keywords. Open channel flow, River flow, Euler equations, Asymptotic approximation, SerreGreen-Naghdi equations, Free surface shallow water equations, Non-hydrostatic pressure, Dispersive.

\section{Introduction}

The modelling of hydrology of catchment basins and rivers holds a central place in environmental sciences, particularly in connection with water availability, urban sewer systems, flood risks and in particular for tsunamis. Indeed, rivers are known to be the tsunami highways. Waves penetrate through rivers much faster inland than the coastal inundation reaches over the ground, and may lead flooding in low-lying areas located several $\mathrm{km}$ away from the coastline [33]. This is important today in understanding and forecasting the impact of climate variability on the human and natural environment. Modelling these processes and predicting the motion of water is a difficult task for which substantial effort has been devoted [13, 16, 28, 33, 35, 37, 38].

One of the most widely used models to describe the channel and river motion of watercourses is the section-averaged free surface model $[4,9,11]$ which is a generalisation of the well-known SaintVenant system (introduced by Adhémar Jean Claude Barré de Saint-Venant in the 19th Century $[8,14])$ :

$$
\begin{cases}\partial_{t} A+\partial_{x} Q & =0 \\ \partial_{t} Q+\partial_{x}\left(\frac{Q^{2}}{A}+I_{1}(x, A)\right) & =I_{2}(x, A) .\end{cases}
$$

\footnotetext{
*Mohamed-Ali-Debyaoui@etud.univ-tln.fr

†Mehmet.Ersoy@univ-tln.fr
} 
In these equations, $A$ is the wet area of fluid cross-section, $Q$ is the water discharge, $I_{1}$ is the hydrostatic pressure and $I_{2}$ is the hydrostatic pressure source term which takes in account of the variation of the section. The Model (1) reduces to the well-known one-dimensional Saint-Venant equations for uniform rectangular section. The free surface model is the first order shallow water approximation of the section-averaged Navier-Stokes or Euler equations under suitable assumptions on the horizontal and the vertical scales (see, e.g., $[4,9,11,14,15]$ and the reference therein).

Thanks to the hyperbolic structure of these equations, sharp transitions between two different flow states result in a discontinuous solution, both in the water surface and in the velocity. These discontinuous solutions (called shocks and also referred as bores) are well-suited to approximate breaking waves with turbulent rollers for large transitions of the Froude's number. However, for small or moderate transitions, the advancing wave front can be followed by a train of freesurface undulations, sometimes called "whelps". This phenomenon, called undular bore (also often called dispersive shock waves), is induced by a non hydrostatic pressure distribution [24]. As a consequence, wave solutions spread out in space as they evolve in time, i.e. waves of different wavelengths travel with different speeds. This is the so-called dispersive effect. Consequently, undular bores are not reproductible with the non-dispersive free surface system and non hydrostatic pressure is required.

Dispersive equations were first introduced by Boussinesq [5] in 1872 to mathematically justify the existence of solitary waves observed by Russell's experiments in 1834. These equations enters in the framework of shallow water equations. They can be obtained as the second order asymptotic approximation in $\mu^{2}$, with $\mu=\left(\frac{H}{L}\right)^{2}$, of the depth-averaged Euler equations where $H$ represents a characteristic water depth and $L$ is a characteristic horizontal scale. The Boussinesq type equations, which are weakly non-linear and weakly dispersive, are defined by an additional small non-linearity parameter $\varepsilon=\frac{a}{H}=O(\mu) \ll 1$ where $a$ is the order of the free surface amplitude. These equations for a flat bottom, for instance in $1 \mathrm{D}$, are given by

$$
\begin{cases}\partial_{t} \xi+\partial_{x}(h \bar{u}) & =O\left(\mu^{2}\right) \\ \partial_{t} \bar{u}+\varepsilon \bar{u} \partial_{x} \bar{u}+\nabla \xi+\mu \mathbb{D} & =O\left(\mu^{2}\right)\end{cases}
$$

where $h$ is the water depth, $\xi$ is the free surface elevation and $\bar{u}$ is the depth-averaged velocity. The term $\mathbb{D}$ represents the dispersive term. In 1877, the KdV equation was discovered by Boussinesq [5] and was later derived by Korteweg and Gustav de Vries (KdV) [18]. This equation approximately describes the evolution of long, one-dimensional waves in many physical settings, including shallowwater waves with weakly non-linear behaviour. This equation can also be obtained in the case of unidirectional waves for which the theoretical framework allows to compute an analytical solution such as 1D solitary wave propagation on flat bottom (see e.g. Boussinesq [2, 5, 18]). We have also the Benjamin, Bona, and Mahony (BBM) equation which is an improvement of the KdV equation [3]. However, in practice and especially in coastal engineering applications, the nearshore wave dynamics being often varying-bottom dependent, dispersive and non-linear, the Boussinesq type equations are not appropriate. In 1967, Peregrine [25] introduced the first weakly non-linear two dimensional Boussinesq type equations for non flat bottom. Witting [36] proposed a method based on Padé expansion to improve the frequency dispersion of the Boussinesq-type equations. From this method, several equations of order $O\left(\mu^{2}\right)$ with improved dispersion characteristic have been proposed, see for instance [22, 23, 29]. In 1953, a one-dimensional, fully non-linear $(\varepsilon=O(1))$ and weakly dispersive equations for flat bottom were derived by Serre [31], independently of $\mathrm{Su}$ and Gardner [32] with

$$
\mathbb{D}(v)=\frac{1}{h} \partial_{x}\left(\frac{h^{3}}{3} \mathcal{D}(v)\right)
$$

where

$$
\mathcal{D}(v)=\left(\partial_{x} v\right)^{2}-\partial_{x} \partial_{t} v-v \partial_{x}^{2} v
$$


This model was then extended to non flat bottom by Seabra-Santos et al. [30], see also [6]. Finally, Green and Naghdi [17] derived the two dimensional fully nonlinear dispersive equations for uneven bottom which are the extension of Serre equations. In the literature, this system is often called SGN equations. Further interesting extensions are also presented in [7, 20, 21, 26, 27, 34] based on either Euler equations, water waves equations or variational principles.

All of the previous dispersive models are obtained either from 3D-2D or 2D-1D reduction, but, up to our knowledge, the 3D-1D reduction has never been done before. Thus, our main goal is to derive from the three-dimensional incompressible and irrotational Euler equations with suitable boundary conditions, a model akin to (2)-(4) via section averaging under the shallow water assumption. The section averaged model that we obtain extends the section-averaged free surface model (1) and the SGN equations (2)-(4):

$$
\left\{\begin{array}{l}
\partial_{t} A+\partial_{x} Q=0 \\
\partial_{t} Q+\partial_{x}\left(\frac{Q^{2}}{A}+I_{1}(x, A)\right)+\mu_{2} \partial_{x}(G(x, A) \mathcal{D}(u))=I_{2}(x, A)+\mathcal{G}(x, A, Q)+O\left(\mu_{2}^{2}\right)
\end{array}\right.
$$

where $A$ is the wet area, $Q=A u$ is the water discharge, $u$ is the section-averaged velocity, $I_{1}$ (resp. $I_{2}$ ) is the hydrostatic pressure (resp. source) term, $G(x, A)$ generalises $\frac{h^{3}}{3}$ in $(3)$, the term $\mathcal{G}(x, A, Q)$ extends the uneven bottom source term, $\mathcal{D}$ is the term given by $(4)$ and therefore $\mathbb{D}=\partial_{x}(G(x, A) \mathcal{D}(u))$ represents the dispersive term.

The paper is organised as follows: in $\S 2$ we present the geometrical settings of the physical domain and the governing equations. In $\S 3$, we obtain the key asymptotic approximation of the horizontal velocity $u$ and the pressure $P$ is decomposed into a hydrostatic $P_{\mathrm{h}}$ and a non-hydrostatic part $P_{\mathrm{nh}}$. Finally, we present in $\S 4$ the derivation of the new section-averaged non-linear dispersive model for arbitrary varying non rectangular open channel/river flows in $\S 4.3$ and for varying rectangular channel in $\S 4.4$.

\section{Notations concerning the physical settings}

- $\sigma(x, z)=\beta(x, z)-\alpha(x, z)$ : width of the section at $z$ with $\beta(x, z)($ resp. $\alpha(x, z))$ is the right (resp. left) boundary point at elevation $z$

- $d(x, y)$ : definition of the boundary of the channel/river cross-section as a function of $y$

- $d^{*}(x)=d\left(x, y^{*}(x)\right)=\min _{y} d(x, y): z$-coordinate of the deepest point of the cross-section

- $\varphi(x, z)=\left\{\begin{array}{l}\alpha(x, z) \text { if } \\ \beta(x, z) \text { otherwise. }\end{array} \quad \varphi(x, z)<y^{*}(x)\right.$ : definition of the boundary of the channel/river cross-section as a function of $z$

- $\Omega(t, x)$ : fluid cross-section

- $\eta(t, x, y): z$-coordinate of the free surface level

- $h(t, x, y)=\eta(t, x, y)-d(x, y)$ : local height of water in $\Omega(t, x)$

- $A(t, x)=|\Omega(t, x)|$ : wet area

- $\boldsymbol{n}_{\mathrm{fs}}$ : outward normal vector to the free surface boundary $\Gamma_{\mathrm{fs}}$ of $\Omega(t, x)$

- $\boldsymbol{n}_{\mathrm{wb}}$ : outward normal vector to the wet boundary $\Gamma_{\mathrm{wb}}$ of $\Omega(t, x)$

\section{Notations concerning the asymptotic parameters}

- $H_{2}$ : characteristic water depth

- $H_{1}$ : characteristic scale of the channel width

- $h_{1}$ : characteristic wave-length in the transversal direction.

- $L$ : characteristic wave-length in the longitudinal direction 
- $\mu_{1}=\frac{h_{1}^{2}}{L^{2}}$ : dispersive parameter in the transversal direction

- $\mu_{2}=\frac{H_{2}^{2}}{L^{2}}$ : usual dispersive parameter

\section{Notations concerning the model}

- $\Omega_{\mathrm{eq}}(t, x)$ : fluid cross-section (flat free surface approximation of $\Omega(t, x)$ )

- $\eta_{\mathrm{eq}}(t, x): z$-coordinate of the free surface level

- $h_{\mathrm{eq}}$ : local height of water in $\Omega_{\mathrm{eq}}(t, x)$

- $A_{\text {eq }}$ : wet area of $\Omega_{\text {eq }}(t, x)$

- $Q_{\text {eq }}$ : discharge

- $P_{\mathrm{h}}$ : hydrostatic pressure

- $P_{\mathrm{nh}}$ : non hydrostatic pressure

\section{Other notations}

- $\langle X\rangle(t, x, z)$ : width-average of the function $(t, x, y, z) \mapsto X(t, x, y, z)$

- $\bar{u}(t, x): \Omega(t, x)$-averaged velocity

- $\bar{u}_{\mathrm{eq}}(t, x): \Omega_{\mathrm{eq}}(t, x)$-averaged velocity

- $X_{q}(t, x, z):=X(t, x, q(x, z), z)$ where $q=\alpha$ or $q=\beta$

- $f_{b}(t, x)=f_{\alpha}\left(t, x, d^{*}(x)\right)$

- $S(x, z)=\int_{d^{*}(x)}^{z} \sigma(x, s) d s$

- $\mathcal{S}(u, x, z)=\frac{1}{\sigma(x, z)} \frac{\partial}{\partial x}(u S(x, z))$

- $\nabla_{k_{1}, k_{2}}(X)$ : gradient of a function $X$ with respect to the variable $k_{1}$ and $k_{2}$

- $\operatorname{div}_{k_{1}, k_{2}}[\boldsymbol{X}]$ : divergence of a vector function $\boldsymbol{X}$ with respect to the variable $k_{1}$ and $k_{2}$

Bold characters are used for vectors notations.

For almost all computations, we assume that $x$ and $t$ are fixed.

\section{The three-dimensional Incompressible Euler equations}

We start in $\S 2.1$ by reviewing the irrotational and incompressible Euler equations in the special geometric setting, describing the physics with a wet boundary on the bottom of the water course and a free surface on the top. Boundary conditions are presented in $§ 2.2$.

\subsection{Geometric set-up and the Euler equations}

Let $T>0$ be an arbitrary time. We consider an incompressible and irrotational fluid moving in the time-space box $[0, T] \times \mathcal{C}$ with typical point $(t,(x, y, z))$ where $\mathcal{C}$ is the geometrical definition of a convex (non-rectangular) channel/river

$$
\mathcal{C}=\left\{(x, y, z) \in \mathbb{R}^{3} ; x \in\left[0, L_{c}\right], \alpha(x, z) \leq y \leq \beta(x, z) \text { and } d(x, y) \leq z\right\}
$$

$L_{c}>0$ is the horizontal length of the domain and for $z \geq d(x, y), \alpha(x, z)$ (respectively $\left.\beta(x, z)\right)$ is the left (respectively right) boundary point at the elevation $z$ as displayed in Fig. 1 . The height of the surface of the water level and the boundary of the section are modelled, respectively, by 
the functions $\eta(t, x, y)$ and $d(x, y)$ with respect to a reference horizontal height $z=0$. For all $x \in\left[0, L_{c}\right]$, we assume that the function $y \mapsto d(x, y)$, has a global minimum at

$$
y^{*}(x) \text { i.e. } d^{*}(x)=d\left(x, y^{*}(x)\right)=\min _{y} d(x, y)
$$

where $y^{*}$ describes the transversal variation of the channel with respect to the main channel/river direction.

We define the local height of the water by

$$
h(t, x, y):=\eta(t, x, y)-d(x, y) .
$$

The boundary of the section can be also described as a function of $y$ by

$$
\forall x \in\left[0, L_{c}\right], \varphi(x, z)=\left\{\begin{array}{l}
\alpha(x, z) \text { if } \\
\beta(x, z) \text { otherwise. }
\end{array}\right.
$$

The width of the section at the elevation $z$ is given by an increasing function

$$
z \mapsto \sigma(x, z)=\beta(x, z)-\alpha(x, z) .
$$

The wet region is defined as the region in which the fluid resides at each time $t \in[0, T]$

$$
\Omega(t)=\bigcup_{0 \leq x \leq L} \Omega(t, x)
$$

with its global counterpart

$$
\Omega:=\bigcup_{0 \leq t \leq T} \Omega(t) .
$$

$\Omega(t, x)$ is the cross-section of fluid at the position $x$ of the channel $\mathcal{C}$ :

$$
\Omega(t, x)=\left\{(y, z) \in \mathbb{R}^{2} ; \alpha(x, z) \leq y \leq \beta(x, z) \text { and } d(x, y) \leq z \leq \eta(t, x, y)\right\}
$$

and $A(t, x)=|\Omega(t, x)|$ is the wet area of the cross-section of fluid:

$A(t, x)=\int_{\Omega(t, x)} d \omega=\int_{y^{-}(t, x)}^{y^{+}(t, x)} \eta(t, x, y)-d(x, y) d y=\int_{d^{*}(x)}^{\max _{y} \eta(t, x, y)} \int_{\alpha(x, z)}^{\beta(x, z)} \mathbb{1}_{\{d(x, y) \leq z \leq \eta(t, x, y)\}} d y d z$

where

$$
y^{-}(t, x):=\min \{y \in \mathbb{R} ; \eta(t, x, y)=d(x, y)\} \text { and } y^{+}(t, x):=\max \{y \in \mathbb{R} ; \eta(t, x, y)=d(x, y)\}
$$

stands for the left and the right transversal limit at $z=\eta$ as shown in Fig. 1.

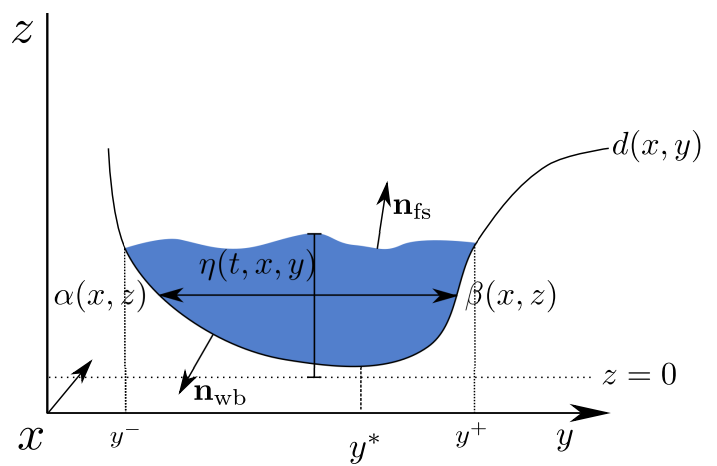

Figure 1: Geometric set-up 
We assume that the flow is governed, on the space-time domain $\Omega$, by the three-dimensional irrotational and incompressible Euler equations

$$
\begin{gathered}
\operatorname{div}\left[\rho_{0} \boldsymbol{u}\right]=0, \\
\frac{\partial}{\partial t}\left(\rho_{0} \boldsymbol{u}\right)+\operatorname{div}\left[\rho_{0} \boldsymbol{u} \otimes \boldsymbol{u}\right]+\nabla p-\rho_{0} \boldsymbol{F}=0
\end{gathered}
$$

where $\boldsymbol{u}=(u, v, w)$ is the velocity field, $\rho_{0}$ is the density of the fluid (taken to be constant), $\boldsymbol{F}=(0,0,-g)$ is the external force of gravity with constant $g$ and $p$ is the pressure.

These equations are completed by the irrotational equation:

$$
\operatorname{curl}[\boldsymbol{u}]=0 \text {. }
$$

\subsection{Boundary conditions}

For a given time $t \in[0, T]$ and $x \in\left[0, L_{c}\right]$, the boundary of the fluid cross-section $\Omega(t, x)$ is composed of a free surface $\Gamma_{\mathrm{fs}}(t, x)=\left\{(y, z) \in \mathbb{R}^{2} ; z=\eta(t, x, y)\right\}$ and a wet boundary (part of the boundary in contact with water) $\Gamma_{\mathrm{wb}}(x)=\left\{(y, z) \in \mathbb{R}^{2} ; z=d(x, y)\right\}$ such that

$$
\partial \Omega(t, x)=\Gamma_{\mathrm{fs}}(t, x) \cup \Gamma_{\mathrm{wb}}(x) .
$$

We prescribe a kinematic boundary condition at the free surface boundary and a no-penetration condition at the wet boundary as described below.

\subsubsection{Free surface boundary conditions}

Assuming a kinematic boundary condition, we set for all points $\boldsymbol{m}(t, x, y)=(x, y, \eta(t, x, y)) \in$ $\Gamma_{\mathrm{fs}}(t, x)$,

$$
\boldsymbol{u} \cdot \boldsymbol{n}_{\mathrm{fs}}=\partial_{t} \boldsymbol{m} \cdot \boldsymbol{n}_{\mathrm{fs}}
$$

where

$$
\boldsymbol{n}_{\mathrm{fs}}(t, x, y)=\frac{1}{\sqrt{1+\left(\partial_{x} \eta(t, x, y)\right)^{2}+\left(\partial_{y} \eta(t, x, y)\right)^{2}}}\left(\begin{array}{c}
-\partial_{x} \eta(t, x, y) \\
-\partial_{y} \eta(t, x, y) \\
1
\end{array}\right)
$$

is the unit outward normal vector to the free surface. This leads to the following explicit form of the kinematic boundary condition

$$
\partial_{t} \eta+u \partial_{x} \eta+v \partial_{y} \eta=w \text { on } z=\eta
$$

We also assume that the pressure at the free surface level is equal to the atmospheric pressure $p_{0}$

$$
p=p_{0} \text { on } z=\eta \text {. }
$$

In the sequel, without loss of generality, we set $p_{0}=0$.

\subsubsection{Wet boundary conditions}

On the wet boundary, i.e., the part of the boundary in contact with water, prescribing a nopenetration condition, we set for all points $\boldsymbol{m}(x, y)=(x, y, d(x, y)) \in \Gamma_{\mathrm{wb}}(x)$,

$$
\boldsymbol{u} \cdot \boldsymbol{n}_{\mathrm{wb}}=0
$$

which leads to the following explicit form:

$$
u \partial_{x} d+v \partial_{y} d=w \text { on } z=d
$$


where

$$
\boldsymbol{n}_{\mathrm{wb}}(x, y)=\frac{1}{\sqrt{1+\left(\partial_{x} d(x, y)\right)^{2}+\left(\partial_{y} d(x, y)\right)^{2}}}\left(\begin{array}{c}
\partial_{x} d(x, y) \\
\partial_{y} d(x, y) \\
-1
\end{array}\right)
$$

is the unit outward normal vector to the wet boundary. In view of the definition of the function $\varphi$, see Eq. (5), the no-penetration condition can be also expressed as a function of $(x, z)$ by

$$
u \partial_{x} \varphi+w \partial_{z} \varphi=v \text { on } y=\varphi
$$

where the unit outward normal vector to the wet boundary is

$$
\boldsymbol{n}_{\mathrm{wb}}(x, z)=\frac{1}{\sqrt{1+\left(\partial_{x} \varphi(x, z)\right)^{2}+\left(\partial_{z} \varphi(x, z)\right)^{2}}}\left(\begin{array}{c}
\partial_{x} \varphi(x, z) \\
-1 \\
\partial_{z} \varphi(x, z)
\end{array}\right) .
$$

In this work, we neglect some physical process arising in river flows: sedimentation, exchange between groundwater flows and subsurface flows, porosities, etc. However, these phenomena can be easily integrated to this work, see for instance [10,12], by considering the following boundary condition $\boldsymbol{u} \cdot \boldsymbol{n}_{\mathrm{wb}}=\left(\partial_{t} \boldsymbol{m}+I\right) \cdot \boldsymbol{n}_{\mathrm{wb}}$ where $\partial_{t} \boldsymbol{m}$ models the evolution in time of the bed and $I$ is the infiltration function. $I$ models the amount of water that leaves $(I>0)$ or enters $(I<0)$ the flow per elementary boundary element.

\section{Width-averaged and depth-averaged asymptotic expansions}

In this section, we present the strategy to derive the section-averaged non-linear dispersive model (see §4). To this end, we first introduce in $\S 3.1$ the dimensionless problem by introducing the classical dispersive parameter $\mu_{2}$. We also introduce a dispersive parameter $\mu_{1}$ but in the transversal direction. In this work, we assume that $\mu_{1}<\mu_{2}$ to obtain the section-averaged dispersive model with respect to $\mu_{2}$ as usually done. Due to the structure of the equations, we cannot obtain the model by a direct section-averaging. We need to develop first suitable asymptotic expansions in two steps, briefly summarized below:

- in $§ 3.2$, the Euler equations (9)-(10) are width-averaged to get the following asymptotic expansion of the horizontal fluid velocity

$$
u(t, x, y, z)=\langle u\rangle(t, x, z)+O\left(\mu_{1}\right)
$$

where $\langle u\rangle$ is the width-averaged velocity.

- in $\S 3.3$, the width-averaged Euler equations allows to obtain the asymptotic expansion of the horizontal width-averaged fluid velocity

$$
\langle u\rangle(t, x, z)=\bar{u}(t, x)+\mu_{2} f(\bar{u}(t, x), \Omega(t, x))+O\left(\mu_{2}^{2}\right)
$$

where $\bar{u}$ is the section-averaged velocity for some function $f$ given later on.

Thus, the asymptotic expansion of $u$ up to order $O\left(\mu_{2}^{2}\right)$, the so-called "motion by slices" (see [14]), can be written as follows:

$$
u(t, x, y, z)=\bar{u}(t, x)+\mu_{2} f(\bar{u}(t, x), \Omega(t, x))+O\left(\mu_{2}^{2}\right) .
$$

Finally, using these asymptotic expansions, we are able in $\S 4$ to section-average the Euler equations (9)-(10) to obtain the new one-dimensional non-linear dispersive equations. 


\subsection{Dimensionless Euler equations}

Let us consider the following scales involved in the wave motion: $L$ a characteristic wave-length in the longitudinal direction, $H_{2}$ a characteristic water depth, $H_{1}$ a characteristic scale of the channel width and $h_{1}$ a characteristic wave-length in the transversal direction. We then define the classical dispersive parameter $\mu_{2}$ (see e.g. [19])

$$
\mu_{2}=\frac{H_{2}^{2}}{L^{2}}
$$

and $\mu_{1}=\frac{h_{1}^{2}}{L^{2}}$ where $\mu_{1}$ is also a dispersive parameter but in the transversal direction.

In the following, we consider the asymptotic regime:

$$
h_{1}<H_{1}=H_{2} \ll L
$$

such that the following inequality holds

$$
\mu_{1}<\mu_{2}^{2} .
$$

Under these assumptions, we get the following ordering:

$$
\mu_{1}^{2}<\frac{\mu_{1}^{2}}{\mu_{2}}<\min \left(\frac{\mu_{1}^{2}}{\mu_{2}^{2}}, \mu_{1} \mu_{2}\right)<\max \left(\frac{\mu_{1}^{2}}{\mu_{2}^{2}}, \mu_{1} \mu_{2}\right)<\mu_{1}<\min \left(\frac{\mu_{1}}{\mu_{2}}, \mu_{2}^{2}\right)<\max \left(\frac{\mu_{1}}{\mu_{2}}, \mu_{2}^{2}\right)<\mu_{2} .
$$

We also introduce $\boldsymbol{U}=\left(U, V=\sqrt{\mu_{1}} U, W=\sqrt{\mu_{2}} U\right)$ the scale of fluid velocity so that $V<W<$ $U$. The time scale is $T=\frac{L}{U}$. We set $P=\frac{p}{\rho_{0}}$ and we define $\mathcal{P}=U^{2}$.

This allows us to introduce the dimensionless quantities of time $\widetilde{t}$, space $(\widetilde{x}, \widetilde{y}, \widetilde{z})$, pressure $\widetilde{P}$, depth $\widetilde{d}$, water elevation $\widetilde{\eta}$ and velocity field $(\widetilde{U}, \widetilde{V}, \widetilde{W})$, via the following scaling relation

$$
\begin{array}{lll}
\widetilde{x}=\frac{x}{L}, & \widetilde{P}=\frac{P}{\mathcal{P}}, & \widetilde{\varphi}=\frac{\varphi}{h_{1}}, \\
\widetilde{y}=\frac{y}{h_{1}}, & \widetilde{u}=\frac{u}{U}, & \widetilde{d}=\frac{d}{H_{2}}, \\
\widetilde{z}=\frac{z}{H_{2}}, & \widetilde{v}=\frac{v}{V}, & \widetilde{\eta}=\frac{\eta}{H_{2}}, \\
\widetilde{t}=\frac{t}{T}, & \widetilde{w}=\frac{w}{W} .
\end{array}
$$

Finally, we define the non-dimensional Froude's number

$$
F_{r}=\frac{U}{\sqrt{g H_{2}}} .
$$

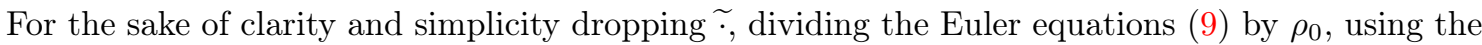
dimensionless variables (15), and reordering the terms with respect to the powers of $\mu_{1}$ and $\mu_{2}$, the dimensionless incompressible Euler system (9) reads as follows:

$$
\begin{aligned}
\partial_{x} u+\partial_{y} v+\partial_{z} w & =0 \\
\partial_{t} u+u \partial_{x} u+v \partial_{y} u+w \partial_{z} u+\partial_{x} P & =0 \\
\mu_{1}\left(\partial_{t} v+u \partial_{x} v+v \partial_{y} v+w \partial_{z} v\right)+\partial_{y} P & =0 \\
\mu_{2}\left(\partial_{t} w+u \partial_{x} w+v \partial_{y} w+w \partial_{z} w\right)+\partial_{z} P & =-\frac{1}{F_{r}{ }^{2}}
\end{aligned}
$$


The boundary conditions (11), (12), (13) and (14) read

$$
\begin{array}{r}
\partial_{t} \eta+u \partial_{x} \eta+v \partial_{y} \eta=w \text { and } P=0 \text { on } z=\eta \\
u \partial_{x} d+v \partial_{y} d=w \text { on } z=d \\
\text { or } u \partial_{x} \varphi+w \partial_{z} \varphi=v \text { on } y=\varphi
\end{array}
$$

and the irrotational equation (10) becomes

$$
\partial_{y} u=\mu_{1} \partial_{x} v, \mu_{1} \partial_{z} v=\mu_{2} \partial_{y} w, \partial_{z} u=\mu_{2} \partial_{x} w .
$$

\subsection{D-2D model reduction and asymptotic expansions}

In this first step, we focus on the width-averaging of System (16)-(19). In particular, we compute the asymptotic expansions of the velocity $(u, v)$ and the pressure $P$ of System (16)-(19) as a function of their width-averages. We obtain what we call "flat free surface approximation" property which means that the variations of the free surface following $y$ can be neglected in the three-dimensional Euler system (16)-(19). Finally, the asymptotic approximation of the width-averaged Euler system is obtained. This averaged model is the starting point for the second step (see §3.3).

\subsubsection{Asymptotic expansions of the fluid velocity}

Given a function $(t, x, y, z) \mapsto X(t, x, y, z)$ and $(x, z) \mapsto q(x, z)$, we define $X_{q}$ as follows

$$
X_{q}(t, x, z):=X(t, x, q(x, z), z)
$$

and we use the notations

$$
\boldsymbol{w}=\left(\begin{array}{c}
u \\
w
\end{array}\right), \operatorname{div}_{x, z}[\boldsymbol{w}]=\partial_{x} u+\partial_{z} w \text { and } \nabla_{x, z}(u)=\left(\begin{array}{c}
\partial_{x} u \\
\partial_{z} u
\end{array}\right)
$$

to represent the vector $\boldsymbol{w}$, the two dimensional divergence operator and the gradient operator with respect to the variable $x$ and $z$.

Integrating the two first equations of the irrotational equations (23) for $s \in[\alpha(x, z), y]$, we get

$$
u(t, x, y, z)=u_{\alpha}(t, x, z)+\mu_{1} \int_{\alpha(x, z)}^{y} \partial_{x} v d s
$$

and

$$
w(t, x, y, z)=w_{\alpha}(t, x, z)+\frac{\mu_{1}}{\mu_{2}} \int_{\alpha(x, z)}^{y} \partial_{z} v d s .
$$

Then, integrating the divergence equation (16) for $s \in[\alpha(x, z), y]$, we obtain

$$
\int_{\alpha(x, z)}^{y} \partial_{y} v d y=-\int_{\alpha(x, z)}^{y} \operatorname{div}_{x, z}[\boldsymbol{w}] d y .
$$

Thanks to Eqs. (24), (25) and (26) and the wet boundary condition (22) for $\varphi=\alpha$ the fluid velocity $v$ is approximated by

$$
v(t, x, y, z)=-\operatorname{div}_{x, z}\left[\boldsymbol{w}_{\alpha}(t, x, z)(y-\alpha(x, z))\right]+O\left(\frac{\mu_{1}}{\mu_{2}}\right) .
$$

Coming back to Eqs. (24) and (25) together with Eq. (27), the fluid velocity $(u, w)$ can be written as

$$
u(t, x, y, z)=u_{\alpha}(t, x, z)-\frac{\mu_{1}}{2} \partial_{x} \operatorname{div}_{x, z}\left[\boldsymbol{w}_{\alpha}(t, x, z)(y-\alpha(x, z))^{2}\right]+O\left(\frac{\mu_{1}^{2}}{\mu_{2}}\right)
$$

and

$$
w(t, x, y, z)=w_{\alpha}(t, x, z)-\frac{\mu_{1}}{2 \mu_{2}} \partial_{z} \operatorname{div}_{x, z}\left[\boldsymbol{w}_{\alpha}(t, x, z)(y-\alpha(x, z))^{2}\right]+O\left(\frac{\mu_{1}^{2}}{\mu_{2}^{2}}\right) .
$$




\subsubsection{Width-averaged Euler equations}

Given a function $(t, x, y, z) \mapsto X(t, x, y, z)$, we define the width-average of $X$ by the quantity

$$
\langle X\rangle(t, x, z):=\frac{1}{\sigma(x, z)} \int_{\alpha(x, z)}^{\beta(x, z)} X(t, x, y, z) d y
$$

where $\sigma(x, z)=\beta(x, z)-\alpha(x, z)$ is the width of the section at the elevation $z$.

We average the equations (16)-(19) for $y \in[\alpha(x, z), \beta(x, z)]$ using Leibniz integral rule, to get the width-averaged Euler system:

$$
\left\{\begin{array}{lll}
\frac{\partial}{\partial x}(\sigma\langle u\rangle)+\frac{\partial}{\partial z}(\sigma\langle w\rangle) & =0 \\
\frac{\partial}{\partial t}(\sigma\langle u\rangle)+\frac{\partial}{\partial x}\left(\sigma\left\langle u^{2}\right\rangle\right)+\frac{\partial}{\partial z}(\sigma\langle u w\rangle)+\frac{\partial}{\partial x}(\sigma\langle P\rangle) & = & P_{\beta} \frac{\partial \beta}{\partial x}-P_{\alpha} \frac{\partial \alpha}{\partial x} \\
\mu_{2}\left(\frac{\partial}{\partial t}(\sigma\langle w\rangle)+\frac{\partial}{\partial x}(\sigma\langle u w\rangle)+\frac{\partial}{\partial z}\left(\sigma\left\langle w^{2}\right\rangle\right)\right)+\frac{\partial}{\partial z}(\sigma\langle P\rangle) & = & -\frac{\sigma}{F_{r}{ }^{2}}+P_{\beta} \frac{\partial \beta}{\partial z}-P_{\alpha} \frac{\partial \alpha}{\partial z} \\
P_{\beta} & =P_{\alpha}-\mu_{1}\left\langle\frac{D v}{D t}\right\rangle
\end{array}\right.
$$

where $\frac{D v}{D t}$ stands for the material derivative

$$
\frac{D v}{D t}:=\partial_{t} v+u \partial_{x} v+v \partial_{y} v+w \partial_{z} v .
$$

Asymptotic expansions of the width-averaged terms in System (30).

Thanks to the expressions (28) and (29), the average of the terms in System (30) can be written

$$
\begin{aligned}
\sigma(x, z)\langle u\rangle(t, x, z)= & \sigma(x, z) u_{\alpha}(t, x, z)-\frac{\mu_{1}}{6} \partial_{x} \operatorname{div}_{x, z}\left[\boldsymbol{w}_{\alpha}(t, x, z) \sigma(x, z)^{3}\right]+O\left(\frac{\mu_{1}^{2}}{\mu_{2}}\right), \\
\sigma(x, z)\langle w\rangle(t, x, z)= & \sigma(x, z) w_{\alpha}(t, x, z)-\frac{\mu_{1}}{6 \mu_{2}} \partial_{z} \operatorname{div}_{x, z}\left[\boldsymbol{w}_{\alpha}(t, x, z) \sigma(x, z)^{3}\right]+O\left(\frac{\mu_{1}^{2}}{\mu_{2}^{2}}\right) \\
\sigma(x, z)\left\langle u^{2}\right\rangle(t, x, z)= & \sigma(x, z) u_{\alpha}^{2}(t, x, z)+O\left(\frac{\mu_{1}^{2}}{\mu_{2}}\right) \\
\sigma(x, z)\left\langle w^{2}\right\rangle(t, x, z)= & \sigma(x, z) w_{\alpha}^{2}(t, x, z)+O\left(\frac{\mu_{1}^{2}}{\mu_{2}^{2}}\right) \\
\sigma(x, z)\langle u w\rangle(t, x, z)= & \sigma(x, z) u_{\alpha}(t, x, z) w_{\alpha}(t, x, z) \\
& -u_{\alpha}(t, x, z) \frac{\mu_{1}}{6 \mu_{2}} \partial_{z} \operatorname{div}_{x, z}\left[\boldsymbol{w}_{\alpha}(t, x, z) \sigma(x, z)^{3}\right]+O\left(\frac{\mu_{1}^{2}}{\mu_{2}^{2}}\right) .
\end{aligned}
$$

\section{Irrotationality.}

We lose the irrotational condition (23) by width-averaging, since we get

$$
\frac{\partial}{\partial z}(\sigma\langle u\rangle)=\mu_{2} \frac{\partial}{\partial x}(\sigma\langle w\rangle)+\left(u_{\beta} \partial_{z} \beta-w_{\beta} \partial_{x} \beta-u_{\alpha} \partial_{z} \alpha+w_{\alpha} \partial_{x} \alpha\right) .
$$

However, using the last equation in (23) together with Eqs. (28) and (29), we obtain

$$
\frac{\partial u_{\alpha}}{\partial z}=\mu_{2} \frac{\partial w_{\alpha}}{\partial x}+O\left(\mu_{1}\right) .
$$

The approximated irrotational condition can be also written as a function of $(\langle u\rangle,\langle w\rangle)$ by means of Eqs. (31) and (32). 


\section{Asymptotic expansion of the pressure.}

The last equation in System (30) allows to write

$$
P_{\beta}=P_{\alpha}+O\left(\mu_{1}\right) .
$$

Thanks to (37), on one hand, the terms in the right hand side of the second and third equations of System (30) can be simplified in

$$
P_{\beta} \nabla_{x, z}(\beta)-P_{\alpha} \nabla_{x, z}(\alpha)=P_{\alpha} \nabla_{x, z}(\sigma)+O\left(\mu_{1}\right) .
$$

On the other hand, coming back to System (16)-(19) together with Eq. (37), integrating Eq. (18) for $s \in[\alpha(x, z), y]$, we show that

$$
P(t, x, y, z)=P_{\alpha}(t, x, z)-\mu_{1} \int_{\alpha(x, z)}^{y} \frac{D v}{D t} d s=P_{\alpha}(t, x, z)+O\left(\mu_{1}\right) .
$$

As a consequence, the width-averaged pressure $\langle P\rangle$ can be approximated by $P_{\alpha}$ at order $O\left(\mu_{1}\right)$, i.e.

$$
\langle P\rangle(t, x, z)=P_{\alpha}(t, x, z)+O\left(\mu_{1}\right) .
$$

\section{Asymptotic expansion of the free surface.}

Using Eqs. (29) and (38) in Eq. (19), we can write the $z$-gradient of the pressure as

$$
\frac{\partial}{\partial z} P_{\alpha}(t, x, z)=-\frac{1}{{F_{r}}^{2}}-\mu_{2} \frac{D}{D t} w_{\alpha}(t, x, z)+O\left(\mu_{1}\right) .
$$

Next, integrating this equation for $s \in[z, \eta(t, x, y)]$ using the boundary condition (20) with Eq. (38) lead to

$$
P_{\alpha}(t, x, z)=\frac{\eta(t, x, y)-z}{F_{r}{ }^{2}}+\mu_{2} \int_{z}^{\eta(t, x, y)} \frac{D}{D t} w_{\alpha}(t, x, s) d s+O\left(\mu_{1}\right) .
$$

Thus, taking the $y$-derivative of this expression yields to

$$
0=\partial_{y} \eta\left(\frac{1}{F_{r}^{2}}+\mu_{2} \frac{D}{D t} w_{\alpha \mid z=\eta}\right)+O\left(\mu_{1}\right)=-\partial_{y} \eta \partial_{z} P_{\mid z=\eta}+O\left(\mu_{1}\right) .
$$

Consequently, since $\partial_{z} P_{\mid z=\eta} \neq 0$, we get $\partial_{y} \eta=O\left(\mu_{1}\right)$, i.e., we obtain the flat surface approximation

$$
\eta(t, x, y)=\eta_{\mathrm{eq}}(t, x)+O\left(\mu_{1}\right)
$$

for some function $\eta_{\mathrm{eq}}$ defined hereafter. It means that one can neglect the $y$-variations of the free surface of the three-dimensional model (16)-(19) (see Fig. 2). In other words, the fluid cross-section $\Omega(t, x)(7)$ and the wet area $A(t, x)(8)$ can be simplified in

$$
\Omega_{\mathrm{eq}}(t, x)=\left\{(y, z) \in \mathbb{R}^{2} ; \alpha(x, z) \leq y \leq \beta(x, z) \text { and } d^{*}(x) \leq z \leq \eta_{\mathrm{eq}}(t, x)\right\}
$$

with

$$
A_{\text {eq }}=\left|\Omega_{\text {eq }}(t, x)\right|
$$


thanks to

$$
\begin{aligned}
A(t, x) & =\int_{\Omega(t, x)} d y d z \\
& =\int_{y^{-}(t, x)}^{y^{+}(t, x)} \eta(t, x, y)-d(x, y) d y \\
& =\int_{y^{-}(t, x)}^{y^{+}(t, x)} \eta_{\mathrm{eq}}(t, x)-d(x, y) d y+O\left(\mu_{1}\right) \\
& =\int_{d^{*}(x)}^{\eta_{\mathrm{eq}}(t, x)} \sigma(x, z) d z+O\left(\mu_{1}\right) \\
& =\int_{\Omega_{\mathrm{eq}}(t, x)} d y d z+O\left(\mu_{1}\right) \\
& =A_{\mathrm{eq}}(t, x)+O\left(\mu_{1}\right)
\end{aligned}
$$

Therefore, in the following, we consider the fluid cross-section $\Omega_{\mathrm{eq}}$ instead of $\Omega$ for which computations are easier. In the following, we set $\eta_{\mathrm{eq}}$ as

$$
\eta_{\mathrm{eq}}(t, x)=\eta\left(t, x, y^{*}(x)\right) .
$$

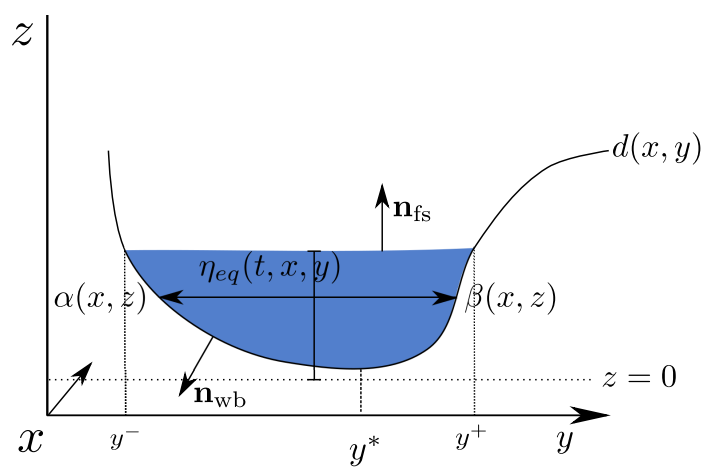

Figure 2: Equivalent geometric set-up

\section{Width-averaged equations.}

Using all the previous equations (31)-(35) and (38)-(39) in System (30), we obtain the widthaveraged Euler equations expressed as the couple of unknown $\left(\boldsymbol{w}_{\alpha}, P_{\alpha}\right)$ :

$$
\begin{aligned}
\operatorname{div}_{x, z}\left[\sigma \boldsymbol{w}_{\alpha}\right]+O\left(\frac{\mu_{1}^{2}}{\mu_{2}^{2}}\right) & =\frac{\mu_{1}}{6 \mu_{2}} \frac{\partial}{\partial z}\left(\sigma \partial_{z}\left(\operatorname{div}_{x, z}\left[\boldsymbol{w}_{\alpha} \sigma^{3}\right]\right)\right) \\
\frac{\partial}{\partial t}\left(\sigma u_{\alpha}\right)+\operatorname{div}_{x, z}\left[\sigma u_{\alpha} \boldsymbol{w}_{\alpha}\right]+\frac{\partial}{\partial x}\left(\sigma P_{\alpha}\right)+O\left(\frac{\mu_{1}^{2}}{\mu_{2}^{2}}\right) & =P_{\alpha} \frac{\partial \sigma}{\partial x}+\frac{\mu_{1}}{6 \mu_{2}} \partial_{x}\left(u_{\alpha} \partial_{z} \operatorname{div}_{x, z}\left[\boldsymbol{w}_{\alpha} \sigma^{3}\right]\right) \\
\mu_{2}\left(\frac{\partial}{\partial t}\left(\sigma w_{\alpha}\right)+\operatorname{div}_{x, z}\left[\sigma w_{\alpha} \boldsymbol{w}_{\alpha}\right]\right)+\frac{\partial}{\partial z}\left(\sigma P_{\alpha}\right) & =-\frac{\sigma}{F_{r}{ }^{2}}+P_{\alpha} \frac{\partial \sigma}{\partial z}+O\left(\mu_{1}\right)
\end{aligned}
$$

where the fluid domain is now defined (see Eqs. (39)-(40) and Fig. 3) by

$$
\langle\Omega\rangle(t, x)=\left\{z \in \mathbb{R} ; d^{*}(x) \leq z \leq \eta_{\mathrm{eq}}(t, x)\right\} .
$$

For a given time $t \in[0, T]$ and $x \in\left[0, L_{c}\right]$, the boundary of the fluid cross-section $\partial\langle\Omega\rangle(t, x)$ is composed of a free surface $\left\langle\Gamma_{\mathrm{fs}}\right\rangle(t, x)=\left\{z \in \mathbb{R} ; z=\eta_{\mathrm{eq}}(t, x)\right\}$ and a wet boundary $\left\langle\Gamma_{\mathrm{wb}}\right\rangle(x)=$ $\left\{z \in \mathbb{R} ; z=d^{*}(x)\right\}$ such that

$$
\partial\langle\Omega\rangle(t, x)=\left\langle\Gamma_{\mathrm{fs}}\right\rangle(t, x) \cup\left\langle\Gamma_{\mathrm{wb}}\right\rangle(x)
$$


The unit outward normal vector to the free surface is

$$
\left\langle\boldsymbol{n}_{\mathrm{fs}}\right\rangle(t, x)=\frac{1}{\sqrt{1+\left(\partial_{x} \eta_{\mathrm{eq}}(t, x)\right)^{2}}}\left(\begin{array}{c}
-\partial_{x} \eta_{\mathrm{eq}}(t, x) \\
1
\end{array}\right)
$$

and the unit outward normal vector to the wet boundary is

$$
\left\langle\boldsymbol{n}_{\mathrm{wb}}\right\rangle(x)=\frac{1}{\sqrt{1+\left(\partial_{x} d^{*}(x)\right)^{2}}}\left(\begin{array}{c}
\partial_{x} d^{*}(x) \\
-1
\end{array}\right) .
$$

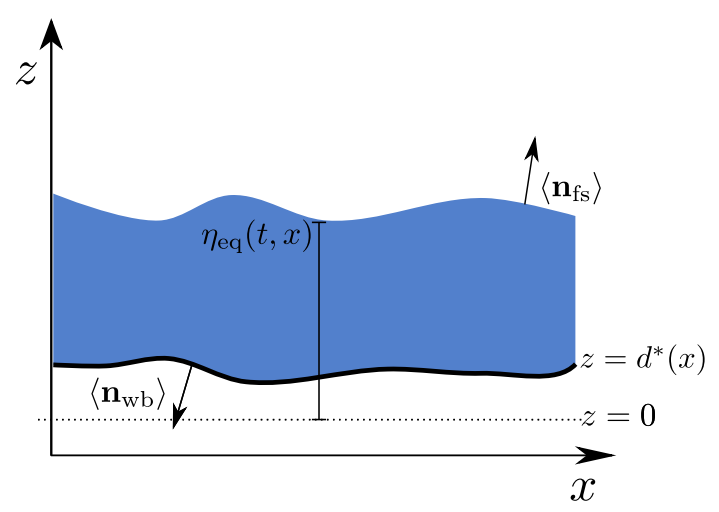

Figure 3: Width-averaged geometric set-up

These equations are completed with the irrotational condition (36) and the following boundary conditions

$$
\begin{array}{r}
\frac{\partial \eta_{\mathrm{eq}}}{\partial t}+u_{\alpha} \frac{\partial \eta_{\mathrm{eq}}}{\partial x}=w_{\alpha}+O\left(\frac{\mu_{1}}{\mu_{2}}\right) \text { and } P_{\alpha}=O\left(\mu_{1}\right) \text { on } z=\eta_{\mathrm{eq}} \\
u_{\alpha} \partial_{x} d=w_{\alpha}+O\left(\frac{\mu_{1}}{\mu_{2}}\right) \text { on } z=d
\end{array}
$$

which are obtained through Eqs. (20) and (21) using Eqs. (27), (28), (29).

These end the first step.

\subsection{D-1D like model reduction and asymptotic expansions}

In what follows, we note $f_{b}(t, x)=f_{\alpha}\left(t, x, d^{*}(x)\right)$ for a given function $f$ and $S$

$$
S(x, z)=\int_{d^{*}(x)}^{z} \sigma(x, s) d s
$$

the wet area of water between $d^{*}(x)$ and $z$.

In the sequel, we consider the fluid cross-section (40) $\Omega_{\mathrm{eq}}(t, x)$ instead of $\Omega(t, x)$ thanks to $(42)$ (see also $(7)$ ). Thus, we define the local height of the water in $\Omega_{\text {eq }}(t, x)$ by

$$
h_{\mathrm{eq}}(t, x, y)=\eta_{\mathrm{eq}}(t, x)-d(x, y) .
$$

In this new setting, the section-average $\bar{f}_{\text {eq }}$ of a function $f$ can be defined by

$$
\bar{f}_{\mathrm{eq}}=\frac{1}{A_{\mathrm{eq}}(t, x)} \int_{d^{*}(x)}^{\eta_{\mathrm{eq}}(t, x)} \int_{\alpha(x, z)}^{\beta(x, z)} f(t, x, y, z) d y d z
$$




\subsubsection{Asymptotic expansion of the fluid velocity}

Let us first integrate Eq. (36) for $s \in\left[d^{*}(x), z\right]$, to get

$$
u_{\alpha}(t, x, z)=u_{b}(t, x)+\mu_{2} \int_{d^{*}(x)}^{z} \frac{\partial w_{\alpha}}{\partial x} d s+O\left(\mu_{1}\right) .
$$

Then, dropping all the lower terms in $O\left(\frac{\mu_{1}}{\mu_{2}}\right)$ in the first equation of System (43):

$$
\frac{\partial}{\partial z}\left(\sigma w_{\alpha}\right)=-\frac{\partial}{\partial x}\left(\sigma u_{\alpha}\right)+O\left(\frac{\mu_{1}}{\mu_{2}}\right)
$$

and integrating the above equation for $s \in\left[d^{*}(x), z\right]$, keeping in mind the boundary condition (45), we obtain

$$
\sigma(x, z) w_{\alpha}(t, x, z)=-\frac{\partial}{\partial x}\left(\int_{d^{*}(x)}^{z} \sigma u_{\alpha} d s\right)+O\left(\frac{\mu_{1}}{\mu_{2}}\right) .
$$

By means of Eq. (46), the previous equation becomes:

$$
w_{\alpha}(t, x, z)=-\frac{1}{\sigma(x, z)} \frac{\partial}{\partial x}\left(u_{b}(t, x) S(x, z)\right)+O\left(\mu_{2}\right) .
$$

By injecting Eq. (47) in (46), we get

$$
u_{\alpha}(t, x, z)=u_{b}(t, x)-\mu_{2} \int_{d^{*}(x)}^{z} \partial_{x} \mathcal{S}\left(u_{b}, x, s\right) d s+O\left(\mu_{2}^{2}\right)
$$

where

$$
\mathcal{S}(u, x, z)=\frac{1}{\sigma(x, z)} \frac{\partial}{\partial x}(u S(x, z))
$$

As a consequence, we have

$$
\begin{aligned}
\bar{u}_{\mathrm{eq}} & =\frac{1}{A_{\mathrm{eq}}(t, x)} \int_{d^{*}(x)}^{\eta_{\mathrm{eq}}(t, x)} \int_{\alpha(x, z)}^{\beta(x, z)} u(t, x, y, z) d y d z \\
& =\frac{1}{A_{\mathrm{eq}}(t, x)} \int_{d^{*}(x)}^{\eta_{\mathrm{eq}}(t, x)} \sigma(x, z)\langle u\rangle(t, x, z) d z \\
& =\frac{1}{A_{\mathrm{eq}}(t, x)} \int_{d^{*}(x)}^{\eta_{\mathrm{eq}}(t, x)} \sigma(x, z) u_{\alpha}(t, x, z) d z+O\left(\mu_{1}\right)
\end{aligned}
$$

and through Eq. (48), we obtain

$$
\begin{aligned}
\bar{u}_{\mathrm{eq}}(t, x) & =\frac{1}{A_{\mathrm{eq}}(t, x)} \int_{d^{*}(x)}^{\eta_{\mathrm{eq}}(t, x)} \sigma(x, z)\left(u_{b}(t, x)-\mu_{2} \int_{d^{*}(x)}^{z} \partial_{x} \mathcal{S}\left(u_{b}, x, s\right) d s\right) d z+O\left(\mu_{2}^{2}\right) \\
& =u_{b}(t, x)-\frac{\mu_{2}}{A_{\mathrm{eq}}(t, x)} \int_{d^{*}(x)}^{\eta_{\mathrm{eq}}(t, x)} \sigma(x, z)\left(\int_{d^{*}(x)}^{z} \partial_{x} \mathcal{S}\left(u_{b}, x, s\right) d s\right) d z+O\left(\mu_{2}^{2}\right)
\end{aligned}
$$

i.e.

$$
u_{b}(t, x)=\bar{u}_{\mathrm{eq}}(t, x)+\frac{\mu_{2}}{A_{\mathrm{eq}}(t, x)} \int_{d^{*}(x)}^{\eta_{\mathrm{eq}}(t, x)} \sigma(x, z)\left(\int_{d^{*}(x)}^{z} \partial_{x} \mathcal{S}\left(u_{b}, x, s\right) d s\right) d z+O\left(\mu_{2}^{2}\right) .
$$

Using the fact that

$$
u_{b}(t, x)=\bar{u}_{\mathrm{eq}}(t, x)+O\left(\mu_{2}\right)
$$


in Eq. (50) allows to write $u_{b}(t, x)$ as a function of $\bar{u}_{\text {eq }}$ :

$$
u_{b}(t, x)=\bar{u}_{\mathrm{eq}}(t, x)+\frac{\mu_{2}}{A_{\mathrm{eq}}(t, x)} \int_{d^{*}(x)}^{\eta_{\mathrm{eq}}(t, x)} \sigma(x, z)\left(\int_{d^{*}(x)}^{z} \partial_{x} \mathcal{S}\left(\bar{u}_{\mathrm{eq}}, x, s\right) d s\right) d z+O\left(\mu_{2}^{2}\right) .
$$

Finally, thanks to (24), (48), (51), by linearity of $\mathcal{S}(49)$, we obtain

$$
\begin{aligned}
u(t, x, y, z) & =u_{\alpha}(t, x, z)+O\left(\mu_{1}\right) \\
& =u_{b}(t, x)-\mu_{2} \int_{d^{*}(x)}^{z} \partial_{x} \mathcal{S}\left(u_{b}, x, s\right) d s+O\left(\mu_{2}^{2}\right) \\
& =\bar{u}_{\mathrm{eq}}(t, x)+\mu_{2} B_{0}\left(\bar{u}_{\mathrm{eq}}, x, z\right)+O\left(\mu_{2}^{2}\right)
\end{aligned}
$$

where

$$
B_{0}\left(\bar{u}_{\mathrm{eq}}, x, z\right)=\frac{1}{A_{\mathrm{eq}}(t, x)} \int_{d^{*}(x)}^{\eta_{\mathrm{eq}}(t, x)}\left(\sigma(x, z) \int_{d^{*}(x)}^{z} \partial_{x} \mathcal{S}\left(\bar{u}_{\mathrm{eq}}, x, s\right) d s\right) d z-\int_{d^{*}(x)}^{z} \partial_{x} \mathcal{S}\left(\bar{u}_{\mathrm{eq}}, x, s\right) d s .
$$

Repeating the computations with Eqs. (29), (47) and (52), we find

$$
w(t, x, y, z)=-\mathcal{S}\left(\bar{u}_{\mathrm{eq}}, x, z\right)+O\left(\frac{\mu_{1}}{\mu_{2}}\right) .
$$

\subsubsection{Pressure decomposition}

Integrating for $s \in\left[z, \eta_{\mathrm{eq}}\right]$, the third equation of System (43), we get

$$
P_{\alpha}(t, x, z)=\frac{\left(\eta_{\mathrm{eq}}(t, x)-z\right)}{F_{r}{ }^{2}}+\int_{z}^{\eta_{\mathrm{eq}}(t, x)} \frac{\mu_{2}}{\sigma}\left(\frac{\partial}{\partial t}\left(\sigma w_{\alpha}\right)+\operatorname{div}_{x, z}\left[\sigma w_{\alpha} \boldsymbol{w}_{\alpha}\right]\right) d s+O\left(\mu_{1}\right) .
$$

Thanks to the free surface boundary condition (44), Eq. (52) and Eq. (54), we obtain the asymptotic expansion of the pressure $P$ at order $O\left(\mu_{2}^{2}\right)$

$$
P(t, x, y, z)=P_{\mathrm{h}}(t, x, z)+\mu_{2} P_{\mathrm{nh}}(t, x, z)+O\left(\mu_{2}^{2}\right)
$$

where

is the usual hydrostatic pressure and

$$
P_{\mathrm{h}}(t, x, z)=\frac{\left(\eta_{\mathrm{eq}}(t, x)-z\right)}{F_{r}^{2}}
$$

$$
\begin{aligned}
P_{\mathrm{nh}}(t, x, z)= & \int_{z}^{\eta_{\mathrm{eq}}(t, x)} \frac{1}{2 \sigma(x, s)^{2}} \partial_{z}\left(\left(\sigma(x, s) \mathcal{S}\left(\bar{u}_{\mathrm{eq}}, x, s\right)\right)^{2}\right) d s \\
& -\int_{z}^{\eta_{\mathrm{eq}}(t, x)} \partial_{t} \mathcal{S}\left(\bar{u}_{\mathrm{eq}}, x, s\right)+\frac{\bar{u}_{\mathrm{eq}}(t, x)}{\sigma(x, s)} \partial_{x}\left(\sigma(x, s) \mathcal{S}\left(\bar{u}_{\mathrm{eq}}, x, s\right)\right) d s
\end{aligned}
$$

is a non-hydrostatic pressure.

Handling the terms in the non-hydrostatic pressure differently, one can write

$$
P_{\mathrm{nh}}(t, x, z)=\mathcal{D}\left(\bar{u}_{\mathrm{eq}}\right) \int_{z}^{\eta_{\mathrm{eq}}} \frac{S(x, s)}{\sigma(x, s)} d s+\int_{z}^{\eta_{\mathrm{eq}}} \mathcal{Q}\left(\bar{u}_{\mathrm{eq}}, S, \sigma\right) d s
$$

where

$$
\mathcal{D}(u)=\left(\partial_{x} u\right)^{2}-\partial_{t} \partial_{x} u-u \partial_{x}^{2} u,
$$

and

$$
\begin{aligned}
\mathcal{Q}(u, S, \sigma)= & \frac{u^{2}}{\sigma(x, s)}\left(\frac{\partial_{x} S(x, s) \partial_{x} \sigma(x, s)}{\sigma(x, s)}-\partial_{x}^{2} S(x, s)\right) \\
& +\partial_{x}\left(\frac{u^{2}}{2}\right) \frac{S(x, s) \partial_{x} \sigma(x, s)}{\sigma(x, s)^{2}}-\left(\partial_{t} u+u \partial_{x} u\right) \frac{\partial_{x} S(x, s)}{\sigma(x, s)}
\end{aligned}
$$

The non-hydrostatic pressure (56) is more suitable to have a formulation akin to the SGN equations (2)-(4). 


\section{A new non-linear dispersive model}

In this section,we assume that the flow is governed, on the space-time domain $\Omega_{\mathrm{eq}}=\bigcup_{0 \leq t \leq 1} \Omega_{\mathrm{eq}}(t)$, instead of $\Omega$ (see Def. (6)), by the three-dimensional incompressible and irrotational Euler equations (16)-(19), (23) where the wet region is defined as the region in which the fluid resides at each time $t \in[0,1], \Omega_{\mathrm{eq}}(t)=\bigcup_{0 \leq x \leq 1} \Omega_{\mathrm{eq}}(t, x)$ with $\Omega_{\mathrm{eq}}(t, x)$ the fluid cross-section defined by $(40)$.

These equations (16)-(19) are completed with the kinematic boundary condition at the free surface $(20) \Gamma_{\mathrm{fs}}(t, x)=\left\{z \in \mathbb{R} ; z=\eta_{\mathrm{eq}}(t, x)\right\}$, and a no-penetration condition on the wet boundary (21) $\Gamma_{\mathrm{wb}}(x)=\left\{(y, z) \in \mathbb{R}^{2} ; z=d(x, y)\right\}$ where

$$
\partial \Omega_{\mathrm{eq}}(t, x)=\Gamma_{\mathrm{fs}}(t, x) \cup \Gamma_{\mathrm{wb}}(x) .
$$

These boundary conditions can be written under the following form:

$$
\int_{\partial \Omega_{\mathrm{eq}}(t, x)}\left(\partial_{t} \boldsymbol{m}+u \partial_{x} \boldsymbol{m}-\boldsymbol{v}\right) \cdot \boldsymbol{n} d s=0 \text { on } \partial \Omega_{\mathrm{eq}}(t, x)
$$

where $\boldsymbol{m}(t, x, y, z)=\left\{\begin{array}{lll}\left(y, \eta_{\mathrm{eq}}(t, x)\right) & \text { if } & \boldsymbol{m} \in \Gamma_{\mathrm{fs}}(t, x) \\ (y, d(x, y)) & \text { if } & \boldsymbol{m} \in \Gamma_{\mathrm{wb}}(t, x)\end{array}\right.$ is a boundary point, $\boldsymbol{n}=\left\{\begin{array}{lll}\boldsymbol{n}_{\mathrm{fs}} & \text { if } & \boldsymbol{m} \in \Gamma_{\mathrm{fs}}(t, x) \\ \boldsymbol{n}_{\mathrm{wb}} & \text { if } & \boldsymbol{m} \in \Gamma_{\mathrm{wb}}(t, x)\end{array}\right.$ stands for the outward unit normal vector in the $\Omega_{\mathrm{eq}}(t, x)$-plane (see Fig. 2) and $\boldsymbol{v}=\left(\begin{array}{c}v \\ w\end{array}\right)$.

To work with the wet region, we introduce its indicator function

$$
\Phi(t, x, y, z):=\mathbb{1}_{\Omega_{\mathrm{eq}}(t, x)} \text { for all } t, x, y, z \in \mathbb{R}
$$

where

$$
\mathbb{1}_{P}:= \begin{cases}1 & \text { if } P \text { is true } \\ 0 & \text { if } P \text { is false. }\end{cases}
$$

The function $\Phi$ is advected by the flow so its material derivative, with respect to the flow $\boldsymbol{u}$, must therefore be zero. Moreover, thanks to the incompressibility condition, $\Phi$ satisfies the following indicator transport equation

$$
\partial_{t} \Phi+\partial_{x}(\Phi u)+\operatorname{div}_{y, z}[\Phi \boldsymbol{v}]=0 \text { on } \Omega_{\mathrm{eq}}(t)
$$

where $\operatorname{div}_{y, z}[\boldsymbol{v}]=\partial_{y} v+\partial_{z} w$ with $\boldsymbol{v}=(v, w)$.

\subsection{Eq. of the conservation of the mass}

Integrating Eq. (60) over the section $\Omega_{\mathrm{eq}}(t, x)$ and using Leibniz integral rule, we get the following conservation of the mass equation

$$
\int_{\Omega_{\mathrm{eq}}(t, x)} \partial_{t} \Phi+\partial_{x}(\Phi u)+\operatorname{div}_{y, z}[\Phi \boldsymbol{v}] d y d z=\partial_{t} A_{\mathrm{eq}}+\partial_{x} Q_{\mathrm{eq}}=0
$$

where $Q_{\text {eq }}$ is the water discharge

$$
Q_{\mathrm{eq}}=A_{\mathrm{eq}} \bar{u}_{\mathrm{eq}}
$$

and $\bar{u}_{\mathrm{eq}}$ is $\Omega_{\mathrm{eq}}(t, x)$ section-averaged velocity given by

$$
\bar{u}_{\mathrm{eq}}=\frac{1}{A_{\mathrm{eq}}(t, x)} \int_{\Omega_{\mathrm{eq}}(t, x)} u(t, x, y, z) d y d z .
$$




\subsection{Eq. of the conservation of the momentum}

In order to get the momentum equation of the section-averaged free surface model, we integrate each terms of (17) along the section $\Omega_{\mathrm{eq}}(t, x)$ as follows:

$$
\int_{\Omega_{\mathrm{eq}}(t, x)} \underbrace{\partial_{t}(u)}_{a_{1}}+\underbrace{\partial_{x}\left(u^{2}\right)}_{a_{2}}+\underbrace{\operatorname{div}_{y, z}[u \boldsymbol{v}]}_{a_{3}}+\underbrace{\partial_{x} P}_{a_{4}} d y d z=0 .
$$

4.2.1 Computation of the term $\int_{\Omega_{\mathrm{eq}}(t, x)} a_{1} d y d z$

We have

$$
\int_{\Omega_{\mathrm{eq}}(t, x)} \partial_{t}(u) d y d z=\partial_{t} \int_{\Omega_{\mathrm{eq}}(t, x)} u d y d z-\int_{\partial \Omega_{\mathrm{eq}}(t, x)} u \partial_{t} \boldsymbol{m} \cdot \boldsymbol{n} d s
$$

4.2.2 Computation of the term $\int_{\Omega_{\mathrm{eq}}(t, x)} a_{2} d y d z$

Thanks to the asymptotic approximation of the term $u(52)$ up to order $O\left(\mu_{2}^{2}\right)$, the non-linear term $u^{2}$ can be written as:

$$
u^{2}(t, x, y, z)=\bar{u}_{\mathrm{eq}}(t, x)^{2}+2 \mu_{2} \bar{u}_{\mathrm{eq}} B_{0}\left(\bar{u}_{\mathrm{eq}}, x, z\right)+O\left(\mu_{2}^{2}\right) .
$$

Therefore, we have

$$
\int_{\Omega_{\mathrm{eq}}(t, x)} \partial_{x}\left(u^{2}\right) d y d z=\partial_{x} \int_{\Omega_{\mathrm{eq}}(t, x)} u^{2} d y d z-\int_{\partial \Omega_{\mathrm{eq}}(t, x)} u^{2} \partial_{x} \boldsymbol{m} \cdot \boldsymbol{n} d s
$$

i.e.

$$
\begin{aligned}
\int_{\Omega_{\mathrm{eq}}(t, x)} \partial_{x}\left(u^{2}\right) d y d z= & \partial_{x}\left(Q_{\mathrm{eq}}{ }^{2} / A_{\mathrm{eq}}\right)+\mu_{2} \partial_{x}\left(\frac{Q_{\mathrm{eq}} B\left(x, Q_{\mathrm{eq}} / A_{\mathrm{eq}}\right)}{A_{\mathrm{eq}}}\right) \\
& -\int_{\partial \Omega_{\mathrm{eq}}(t, x)} u^{2} \partial_{x} \boldsymbol{m} \cdot \boldsymbol{n} d s+O\left(\mu_{2}^{2}\right)
\end{aligned}
$$

where $B$ stands for the quadratic part of the fluid velocity, defined via $B_{0}(53)$, and

$$
B\left(x, Q_{\mathrm{eq}} / A_{\mathrm{eq}}\right)=\int_{\Omega_{\mathrm{eq}}(t, x)} 2 B_{0}\left(Q_{\mathrm{eq}} / A_{\mathrm{eq}}, x, z\right) d y d z=2 \int_{d^{*}(x)}^{\eta_{\mathrm{eq}}(t, x)} \sigma(x, z) B_{0}\left(Q_{\mathrm{eq}} / A_{\mathrm{eq}}, x, z\right) d z=0 .
$$

4.2.3 Computation of the term $\int_{\Omega_{\mathrm{eq}}(t, x)} a_{3} d y d z$

We have

$$
\int_{\Omega_{\mathrm{eq}}(t, x)} \operatorname{div}_{y, z}[u \boldsymbol{v}] d y d z=\int_{\partial \Omega_{\mathrm{eq}}(t, x)} u \boldsymbol{v} \cdot \boldsymbol{n} d s
$$

\subsubsection{Computation of the term $\int_{\Omega_{\mathrm{eq}}(t, x)} a_{1}+a_{2}+a_{3} d y d z$}

Gathering the results of the computations (63), (64) and (65), using the boundary conditions (59), we get

$$
\begin{aligned}
\int_{\Omega_{\mathrm{eq}}(t, x)} a_{1}+a_{2}+a_{3} d y d z= & \partial_{t} Q_{\mathrm{eq}}+\partial_{x}\left(Q_{\mathrm{eq}}{ }^{2} / A_{\mathrm{eq}}\right)+\mu_{2} \partial_{x}\left(\frac{Q_{\mathrm{eq}} B\left(x, Q_{\mathrm{eq}} / A_{\mathrm{eq}}\right)}{A_{\mathrm{eq}}}\right) \\
& -\int_{\partial \Omega_{\mathrm{eq}}(t, x)} u\left(\partial_{t} \boldsymbol{m}+u \partial_{x} \boldsymbol{m}-\boldsymbol{v}\right) \cdot \boldsymbol{n} d s+O\left(\mu_{2}^{2}\right) \\
= & \partial_{t} Q_{\mathrm{eq}}+\partial_{x}\left(Q_{\mathrm{eq}}{ }^{2} / A_{\mathrm{eq}}\right)+O\left(\mu_{2}^{2}\right) .
\end{aligned}
$$




\subsubsection{Computation of the term $\int_{\Omega_{\mathrm{eq}}(t, x)} a_{4} d y d z$}

We apply the Leibniz rule to the gradient of the pressure and we obtain

$$
\int_{\Omega_{\mathrm{eq}}(t, x)} \partial_{x} P d y d z=\partial_{x} \int_{\Omega_{\mathrm{eq}}(t, x)} P d y d z-\int_{\partial \Omega_{\mathrm{eq}}(t, x)} P \partial_{x} \boldsymbol{m} \cdot \boldsymbol{n} d s
$$

where

$$
\int_{\partial \Omega_{\mathrm{eq}}(t, x)} P \partial_{x} \boldsymbol{m} \cdot \boldsymbol{n} d s=\int_{\Gamma_{\mathrm{wb}}(x)} P \partial_{x} \boldsymbol{m} \cdot \boldsymbol{n} d s
$$

thanks to the free surface boundary condition (12).

To compute the above boundary integral, we consider the parametrisation

$$
z \in\left[d^{*}(x), \eta_{\mathrm{eq}}(t, x)\right] \mapsto \boldsymbol{m}(x, z)=\left(\begin{array}{c}
\varphi(x, z) \\
z
\end{array}\right)
$$

where $\varphi$ is given by (5). The unit speed curve parameterisation is

$$
\left\|\partial_{y} \boldsymbol{m}(x, z)\right\|=\sqrt{1+\left(\partial_{y} \varphi(x, z)\right)^{2}} .
$$

The outward unit normal is given by

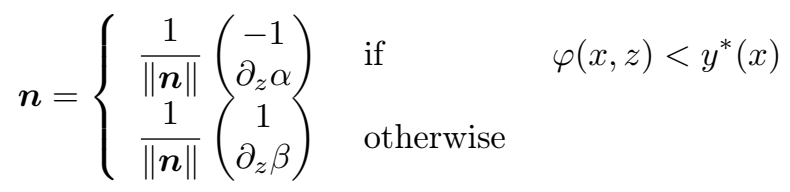

where

$$
\|\boldsymbol{n}(x, z)\|=\left\|\partial_{y} \boldsymbol{m}(x, z)\right\|
$$

Thus, we obtain

$$
\int_{\Gamma_{\mathrm{wb}}(x)} P \partial_{x} \boldsymbol{m} \cdot \boldsymbol{n} d s=\int_{d^{*}(x)}^{\eta_{\mathrm{eq}}(t, x)} P(t, x, z) \partial_{x} \sigma(x, z) d z .
$$

Hydrostatic part: (see Eq. (55)) Using $P=P_{\mathrm{h}}$ in Eq. (67), we get:

$$
\int_{\Omega_{\mathrm{eq}}(t, x)} \partial_{x} P_{\mathrm{h}}(t, x, z) d y d z=\partial_{x}\left(I_{1}(t, x)\right)-I_{2}(t, x)
$$

where

$$
I_{1}(t, x)=\int_{\Omega_{\mathrm{eq}}(t, x)} P_{\mathrm{h}}(t, x, z) d y d z=\int_{d^{*}(x)}^{\eta_{\mathrm{eq}}(t, x)} \frac{\left(\eta_{\mathrm{eq}}(t, x)-z\right)}{F_{r}{ }^{2}} \sigma(x, z) d z
$$

and

$$
I_{2}(t, x)=\int_{d^{*}(x)}^{\eta_{\mathrm{eq}}(t, x)} \frac{\left(\eta_{\mathrm{eq}}(t, x)-z\right)}{F_{r}{ }^{2}} \partial_{x} \sigma(x, z) d z
$$

are respectively the hydrostatic pressure term and the hydrostatic pressure source term.

Non-hydrostatic part: (see Eq. (56)) Using $P=P_{\mathrm{nh}}$ in Eq. (67), we get

$$
\int_{\Omega_{\mathrm{eq}}(t, x)} \partial_{x} P_{\mathrm{nh}}(t, x, z) d y d z=\partial_{x}\left(D I_{1}(t, x)\right)-D I_{2}(t, x)
$$


where

$$
\begin{aligned}
D I_{1}(t, x)= & \int_{d^{*}(x)}^{\eta_{\mathrm{eq}}(t, x)} P_{\mathrm{nh}}(t, x, z) \sigma(x, z) d z \\
= & G\left(x, A_{\mathrm{eq}}(t, x)\right) \mathcal{D}\left(\bar{u}_{\mathrm{eq}}(t, x)\right) \\
& +\int_{d^{*}(x)}^{\eta_{\mathrm{eq}}} \sigma(x, z) \int_{z}^{\eta_{\mathrm{eq}}} \mathcal{Q}\left(\bar{u}_{\mathrm{eq}}(t, x), S(x, s), \sigma\right) d s d z
\end{aligned}
$$

where

$$
G\left(x, A_{\mathrm{eq}}\right)=\int_{d^{*}(x)}^{\eta_{\mathrm{eq}}} \sigma(x, z) \int_{z}^{\eta_{\mathrm{eq}}} \frac{S(x, s)}{\sigma(x, s)} d s d z .
$$

The quantities $\mathcal{D}$ and $\mathcal{Q}$ are given by (57), (58), and

$$
D I_{2}(t, x)=\int_{d^{*}(x)}^{\eta_{\mathrm{eq}}(t, x)} P_{\mathrm{nh}}(t, x, z) \partial_{x} \sigma(x, z) d z
$$

Each term $D I_{j}$ for $j=1,2$ is the non-hydrostatic counterpart of the term $I_{j}$ for $j=1,2$.

Remark 1. The terms $I_{j}(t, x)$ and $D I_{j}(t, x)$, respectively, can be written $I_{j}\left(x, A_{\mathrm{eq}}(t, x)\right)$ and $D I_{j}\left(x, A_{\mathrm{eq}}(t, x), Q_{\mathrm{eq}}(t, x)\right.$ for $j=1$ and $j=2$. In what follows, we make use of these notations.

Finally, gathering Eq. (68) and Eq. (71), noting

$$
\mathcal{G}\left(x, A_{\mathrm{eq}}, Q_{\mathrm{eq}}\right)=-\partial_{x}\left(\int_{d^{*}(x)}^{\eta_{\mathrm{eq}}} \sigma(x, z) \int_{z}^{\eta_{\mathrm{eq}}} \mathcal{Q}\left(\frac{Q_{\mathrm{eq}}}{A_{\mathrm{eq}}}, S, \sigma\right) d s d z\right)+D I_{2}
$$

we obtain

$$
\begin{aligned}
\int_{\Omega_{\mathrm{eq}}(t, x)} \partial_{x} P d y d z= & \partial_{x}\left(I_{1}\left(x, A_{\mathrm{eq}}\right)\right)-I_{2}\left(x, A_{\mathrm{eq}}\right) \\
& +\mu_{2}\left(\partial_{x}\left(G\left(x, A_{\mathrm{eq}}\right) \mathcal{D}\left(\bar{u}_{\mathrm{eq}}\right)\right)-\mathcal{G}\left(x, A_{\mathrm{eq}}, Q_{\mathrm{eq}}\right)\right)
\end{aligned}
$$

Gathering results (66) and (75), we get the equation of the conservation of the momentum.

\subsection{The dispersive model for arbitrary non rectangular channel/river}

From now on, we omit the notations $X_{\text {eq }}$ and $\bar{X}$ for the sake of readability.

Gathering Eqs. (61),(66) and (75), we present the new one-dimensional dispersive model for open channel/river flows

$$
\left\{\begin{array}{l}
\partial_{t} A+\partial_{x} Q=0 \\
\partial_{t} Q+\partial_{x}\left(\frac{Q^{2}}{A}+I_{1}(x, A)\right)+\mu_{2} \partial_{x}(G(x, A) \mathcal{D}(u))=I_{2}(x, A)+\mu_{2} \mathcal{G}(x, A, Q)+O\left(\mu_{2}^{2}\right)
\end{array}\right.
$$

where $A$ is the wet area (41), $Q$ is the water discharge (62), $I_{1}$ (resp. $I_{2}$ ) is the hydrostatic pressure (resp. source) terms (69) (resp. (70)), G(x,A) (72) generalises $\frac{h^{3}}{3}$ in the classical SGN equations (see (3)), $\mathcal{G}(x, A, Q)(74)$ extends the uneven bottom source term in the classical SGN equations and $\partial_{x}(G(x, A) \mathcal{D}(u))$ is the dispersive term where $\mathcal{D}$ is given by $(57)$.

The new section-averaged model extends the section-averaged free surface model for open channel flows $[4,10]$ by taking $\mu_{2}=0$ and extends the SGN equations for uneven bottom $[1,17,30,31]$ to arbitrary channel/river section. 


\subsection{The dispersive model for rectangular section}

For the specific case of the rectangular section, almost all the previous computations are the same. The changes are mainly in the geometrical definition and the boundaries of the channel.

We consider the motion of an incompressible and irrotational fluid with constant density $\rho_{0}>0$ in a three dimensional domain (see Fig. 4)

$$
\Omega(t)=\left\{(x, y, z) \in \mathbb{R}^{3} ; x \in\left[0, L_{c}\right], \alpha(x) \leq y-y^{*}(x) \leq \beta(x), d(x) \leq z \leq \eta(t, x)\right\}
$$

where $y^{*}(x)$ describes the transversal variation of the channel with respect to the main channel direction, defined by

$$
y^{*}(x)=\frac{\alpha(x)+\beta(x)}{2}
$$

where $\alpha$ and $\beta$ are the transversal limit of the channel. Here the bottom $d$ is now a function of $x$ only and $d^{*}(x)=d(x)$ by definition. Thus, in view of the definition of the fluid domain, since $\sigma=\sigma(x)=\beta(x)-\alpha(x)$ and $y^{-}(x)=\alpha(x)$ and $y^{+}(x)=\beta(x)$, the wet-area can be simply defined by

$$
A(t, x)=\sigma(x)\langle h(t, x)\rangle
$$

where $\langle h(t, x)\rangle$ is width-averaged of the local height of the water $h(t, x, y):=\eta(t, x, y)-d(x)$, i.e., $\langle h(t, x)\rangle=\langle\eta(t, x)\rangle-d(x)$.

The boundary of the domain $\Omega(t)$ is defined by $\partial \Omega(t)$ and is decomposed into four parts: the free surface $\Gamma_{\mathrm{fs}}(t)$, the wet boundary $\Gamma_{\mathrm{wb}}(t)$, the inflow boundary $\Gamma_{\mathrm{i}}(t)$ and the ouflow boundary $\Gamma_{\mathrm{o}}(t)$. The wet boundary can be decomposed itself in three parts: the bottom $\Gamma_{\mathrm{b}}(t)$, the left lateral boundary $\Gamma_{\mathrm{lb}}(t)$ and the right one $\Gamma_{\mathrm{rb}}(t)$.

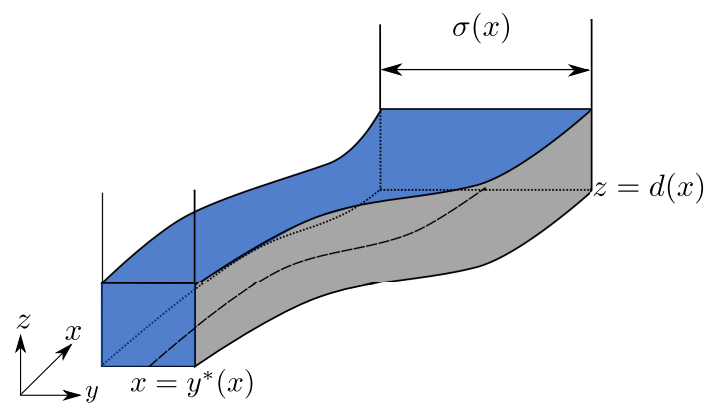

Figure 4: Geometric set-up

The kinematic free surface condition and the wet boundary condition are the same, except the definition of the outward unit normal vector which is now

$$
\boldsymbol{n}_{\mathrm{wb}}=\left\{\begin{array}{lll}
\frac{1}{\sqrt{1+\left(\partial_{x} d\right)^{2}}}\left(\partial_{x} d, 0,-1\right)^{T} & \text { if } \quad \boldsymbol{n}_{\mathrm{wb}}=\boldsymbol{n}_{\mathrm{b}} \\
\frac{1}{\sqrt{1+\left(\partial_{x} \alpha\right)^{2}}}\left(\partial_{x} \alpha,-1,0\right)^{T} & \text { if } & \boldsymbol{n}_{\mathrm{wb}}=\boldsymbol{n}_{\mathrm{lb}} \\
\frac{1}{\sqrt{1+\left(\partial_{x} \beta\right)^{2}}}\left(\partial_{x} \beta, 1,0\right)^{T} & \text { if } & \boldsymbol{n}_{\mathrm{wb}}=\boldsymbol{n}_{\mathrm{rb}}
\end{array}\right.
$$

Thus, we have now

$$
\begin{array}{lll}
u \partial_{x} d-w=0 & \text { on } \Gamma_{\mathrm{b}}(t) \\
u \partial_{x} \alpha-v=0 & \text { on } \Gamma_{\mathrm{lb}}(t) \\
u \partial_{x} \beta+v=0 & \text { on } \Gamma_{\mathrm{rb}}(t)
\end{array}
$$


Almost all the computations are the same to the exception of the computation of the $\int_{\Omega_{\mathrm{eq}}(t, x)} a_{4} d y d z$ which yields to different expression of the hydrostatic pressure source term (70) and the non hydrostatic pressure source term (73). Indeed, since we have now $d^{*}(x)=d(x)$ and $\sigma=\sigma(x)$, thus $A_{\text {eq }}(t, x)=\sigma(x) h_{\text {eq }}(t, x)$. Applying successively the one-dimensional Leibniz integral rule in the $y$-direction and in the $z$-direction, we obtain

$$
\begin{aligned}
& \int_{\Omega_{\mathrm{eq}}(t, x)} \partial_{x} P d y d z=\partial_{x}\left(\int_{d(x)}^{\eta_{\mathrm{eq}}(t, x)} \sigma(x) P(t, x, z) d z\right) \\
& -\int_{d(x)}^{\eta_{\mathrm{eq}}(t, x)} P(t, x, z) \sigma^{\prime}(x) d z+\sigma(x) P(t, x, d(x)) d^{\prime}(x) .
\end{aligned}
$$

Thus, in contrast with the non-rectangular case, the hydrostatic (70) and the non-hydrostatic (73) pressure source terms read

$$
I_{2}(t, x)=\sigma^{\prime}(x) \frac{h_{\mathrm{eq}}(t, x)^{2}}{2 F_{r}{ }^{2}}-\sigma(x) \frac{h_{\mathrm{eq}}(t, x)}{{F_{r}}^{2}} d^{\prime}(x)
$$

and

$$
D I_{2}(t, x)=\int_{d(x)}^{\eta_{\mathrm{eq}}(t, x)} P_{\mathrm{nh}}(t, x, z) \sigma^{\prime}(x) d z-\sigma(x) P_{\mathrm{nh}}(t, x, d(x)) d^{\prime}(x) .
$$

Thus, the model (76) is the same as before but with the formula (77) and (78) instead of (70) and (73).

\section{Conclusion}

In this paper, we have proposed to derive the first section-averaged non-linear dispersive model for open channel and river flows. These equations generalise both the classical section-averaged free surface model and the well-known SGN equations to arbitrary section. The next steps will concern the study of theoretical properties of the model and the numerical approximation for practical applications.

\section{Acknowledgement}

The authors thanks Professor C. Galusinski and the referees for their valuable remarks which led to substantial improvement of the first version of this paper. The second author wishes to grateful Dr Griggio for her help throughout this work and would like to say sincerely "Sağol".

\section{References}

[1] E. Barthélemy. Nonlinear shallow water theories for coastal waves. Surveys in Geophysics, 25(3-4):315-337, 2004.

[2] D. J. Bayraktar and S. L. Beji. A new numerical scheme for improved boussinesq equations with surface pressure. In Sustainable Maritime Transportation and Exploitation of Sea Resources, volume 847, pages 847-854. ROUTLEDGE in association with GSE Research, 2011.

[3] T. B. Benjamin. The stability of solitary waves. Proceedings of the Royal Society of London. A. Mathematical and Physical Sciences, 328(1573):153-183, 1972. 
[4] C. Bourdarias, M. Ersoy, and S. Gerbi. A mathematical model for unsteady mixed flows in closed water pipes. Science China Mathematics, 55(2):221-244, Feb. 2012.

[5] J. Boussinesq. Théorie de l'intumescence liquide appelée onde solitaire ou de translation se propageant dans un canal rectangulaire. Comptes Rendus Acad. Sci (Paris), 72:755-759, 1871.

[6] R. Cienfuegos, E. Barthélemy, and P. Bonneton. A fourth-order compact finite volume scheme for fully nonlinear and weakly dispersive boussinesq-type equations. part ii: boundary conditions and validation. International Journal for Numerical Methods in Fluids, 53(9):1423-1455, 2007.

[7] D. Clamond, D. Dutykh, and D. Mitsotakis. Conservative modified serre-green-naghdi equations with improved dispersion characteristics. Communications in Nonlinear Science and Numerical Simulation, 45:245-257, 2017.

[8] A.-J.-C. B. de Saint-Venant. Théorie du mouvement non-permanent des eaux, avec application aux crues des rivières et à l'introduction des marées dans leur lit. Comptes rendus hebdomadaires des séances de l'Académie des sciences, 73:147-154, 1871.

[9] A. Decoene, L. Bonaventura, E. Miglio, and F. Saleri. Asymptotic derivation of the sectionaveraged shallow water equations for natural river hydraulics. Mathematical Models and Methods in Applied Sciences, 19(03):387-417, 2009.

[10] M. Ersoy. Modeling, mathematical and numerical analysis of various compressible or incompressible flows in thin layer. Theses, Université de Savoie, Sept. 2010.

[11] M. Ersoy. Dimension reduction for incompressible pipe and open channel flow including friction. In Conference Applications of Mathematics 2015, in honor of the 90th birthday of Ivo Babuška and 85th birthday of Milan Práger and Emil Vitásek, pages 17-33, Prague, France, Nov. 2015. J. Brandts and S. Korotov and M. Krizek and K. Segeth and J. Sistek and T. Vejchodsky.

[12] M. Ersoy, O. Lakkis, and P. Townsend. A Saint-Venant shallow water model for overland flows with precipitation and recharge. working paper or preprint, July 2016.

[13] M. Esteves, X. Faucher, S. Galle, and M. Vauclin. Overland flow and infiltration modelling for small plots during unsteady rain: numerical results versus observed values. Journal of hydrology, 228(3):265-282, 2000.

[14] J.-F. Gerbeau and B. Perthame. Derivation of viscous Saint-Venant system for laminar shallow water; numerical validation. Discrete Cont. Dyn. Syst. Ser. B, 1(1):89-102, 2001.

[15] N. Gouta and F. Maurel. A finite volume solver for 1d shallow-water equations applied to an actual river. International Journal for Numerical Methods in Fluids, 38(1):1-19, 2002.

[16] R. Grace and P. S. Eagleson. The modeling of overland flow. Water Resources Research, 2(3):393-403, 1966.

[17] A. E. Green and P. M. Naghdi. A derivation of equations for wave propagation in water of variable depth. Journal of Fluid Mechanics, 78(02):237-246, 1976.

[18] D. J. Korteweg and G. De Vries. Xli. on the change of form of long waves advancing in a rectangular canal, and on a new type of long stationary waves. The London, Edinburgh, and Dublin Philosophical Magazine and Journal of Science, 39(240):422-443, 1895.

[19] D. Lannes. The water waves problem: mathematical analysis and asymptotics, volume 188. American Mathematical Soc., 2013. 
[20] D. Lannes and P. Bonneton. Derivation of asymptotic two-dimensional time-dependent equations for surface water wave propagation. Physics of Fluids (1994-present), 21(1):016601, 2009.

[21] D. Lannes and F. Marche. A new class of fully nonlinear and weakly dispersive green-naghdi models for efficient 2d simulations. Journal of Computational Physics, 282:238-268, 2015.

[22] P. A. Madsen and O. R. Sørensen. A new form of the boussinesq equations with improved linear dispersion characteristics. part 2. a slowly-varying bathymetry. Coastal engineering, 18(3-4):183-204, 1992.

[23] O. Nwogu. Alternative form of boussinesq equations for nearshore wave propagation. Journal of waterway, port, coastal, and ocean engineering, 119(6):618-638, 1993.

[24] D. Peregrine. Calculations of the development of an undular bore. Journal of Fluid Mechanics, 25(2):321-330, 1966.

[25] D. H. Peregrine. Long waves on a beach. Journal of fluid mechanics, 27(4):815-827, 1967.

[26] K. Pons. Modélisation des tsunamis : Propagation et impact. PhD thesis, Université de Toulon, 2018.

[27] G. Richard and S. Gavrilyuk. Modelling turbulence generation in solitary waves on shear shallow water flows. Journal of Fluid Mechanics, 773:49-74, 2015.

[28] M. Rousseau, O. Cerdan, A. Ern, O. Le Maitre, and P. Sochala. Study of overland flow with uncertain infiltration using stochastic tools. Advances in Water Resources, 38:1-12, 2012.

[29] H. A. Schäffer and P. A. Madsen. Further enhancements of boussinesq-type equations. Coastal Engineering, 26(1-2):1-14, 1995.

[30] F. J. Seabra-Santos, D. P. Renouard, and A. M. Temperville. Numerical and experimental study of the transformation of a solitary wave over a shelf or isolated obstacle. Journal of Fluid Mechanics, 176:117-134, 1987.

[31] F. Serre. Contribution à l'étude des écoulements permanents et variables dans les canaux. La Houille Blanche, (6):830-872, 1953.

[32] C. H. Su and C. S. Gardner. Korteweg-de vries equation and generalizations. iii. derivation of the korteweg-de vries equation and burgers equation. Journal of Mathematical Physics, 10(3):536-539, 1969.

[33] Y. Tsuji, T. Yanuma, I. Murata, and C. Fujiwara. Tsunami ascending in rivers as an undular bore. Natural Hazards, 4(2-3):257-266, 1991.

[34] G. Wei, J. T. Kirby, S. T. Grilli, and R. Subramanya. A fully nonlinear boussinesq model for surface waves. part 1. highly nonlinear unsteady waves. Journal of Fluid Mechanics, 294:71-92, 1995.

[35] S. Weill, E. Mouche, and J. Patin. A generalized Richards equation for surface/subsurface flow modelling. Journal of Hydrology, 366(1):9-20, 2009.

[36] J. M. Witting. A unified model for the evolution nonlinear water waves. Journal of Computational Physics, 56(2):203-236, 1984.

[37] D. A. Woolhiser and J. A. Liggett. Unsteady, one-dimensional flow over a plane - the rising hydrograph. Water Resources Research, 3(3):753-771, 1967.

[38] W. Zhang and T. W. Cundy. Modeling of two-dimensional overland flow. Water Resources Research, 25(9):2019-2035, 1989. 